\title{
Un intervalo intergestacional menor a seis meses se asocia con mayor riesgo de parto pretérmino y muerte neonatal
}

\section{Objetivo}

Determinar la relación entre los intervalos intergestacionales (IG) cortos y resultados perinatales adversos y si el IG es un factor de riesgo independiente para un resultado obstétrico desfavorable.

\section{Diseño}

Estudio de cohorte* retrospectivo.

\section{Lugar}

Datos secundarios provenientes del Registro Escocés de Morbilidad, que colecta información de todos los nacimientos ocurridos en dicho país.

\section{Pacientes}

Se incluyeron 89.143 nacimientos ocurridos entre 1992-8 en mujeres secundíparas que hubieran tenido su primer hijo hasta 5 años antes.

\section{Evaluación de factores pronósticos}

Restricción del crecimiento intrauterino, prematurez extrema (24-32 semanas), prematurez moderada (33-36 semanas) y muerte neonatal. Se consideraron intervalos cortos a aquellos menores de seis meses.

\section{Resultados principales}

La frecuencia de IG cortos fue del 5.4\%. En esta muestra, las mujeres con IG corto presentaron una mayor frecuencia de complicaciones obstétricas en la gestación anterior y un perfil sociodemográfico de riesgo para resultados adversos perinatales. Ajustando* por las variables edad materna, estado civil, nivel socioeconómico, uso de tabaco, peso del nacido previo y cesárea previa el IG corto se asoció a un incremento en la prematurez extrema (OR 2,2; IC95\% 1,3 - 3,6) prematurez moderada (OR 1,6; IC95\% 1,3 - 2,0) y muerte neonatal (OR 3,6 IC95\% 1,2 - 10,7). Si el IG pudiese extenderse a más de seis meses, la fracción prevenible en la población ${ }^{*}$ seria de $6,1 \%$ para prematurez extrema; de $3,9 \%$ para prematurez moderada; y de $13,8 \%$ para la muerte neonatal.

Tabla.Relación entre IG y resultados del segundo embarazo. Los OR se calcularon con un intervalo de confianza del $95 \%$.

\begin{tabular}{|c|c|c|c|c|c|}
\hline Resultado & $\begin{array}{l}\text { IG } 1-5 \\
\text { meses }\end{array}$ & $\begin{array}{l}\text { IG 6-11 } \\
\text { meses }\end{array}$ & $\begin{array}{l}\text { IG 12-17 } \\
\text { meses }\end{array}$ & $\begin{array}{l}\text { IG } 18-23 \\
\text { meses }^{\circ}\end{array}$ & $\begin{array}{c}\text { IG } 24-59 \\
\text { meses }\end{array}$ \\
\hline Prematurez extrema & OR 2,2 & OR 1,4 & OR 1,2 & - & OR 1,1 \\
\hline & $(1,4-3,6)$ & $(0,9-2,2)$ & $(0,8-1,7)$ & - & $(0,8-1,6)$ \\
\hline Prematurez moderada & $\begin{array}{c}\text { OR } 1,6 \\
(1,3-2,0)\end{array}$ & $\begin{array}{c}\text { OR } 1,1 \\
(0,9-1,3)\end{array}$ & $\begin{array}{c}\text { OR } 1,1 \\
(0,9-1,3)\end{array}$ & - & $\begin{array}{c}\text { OR } 1,2 \\
(1,0-1,3)\end{array}$ \\
\hline Muerte neonatal & $\begin{array}{c}\text { OR } 3,6 \\
(1,2-10,7)\end{array}$ & $\begin{array}{l}\text { OR } 1,9 \\
(0,7-5,2)\end{array}$ & $\begin{array}{c}\text { OR } 0,3 \\
(0,1-1,5)\end{array}$ & - & $\begin{array}{l}\text { OR } 0,2 \\
(0,5-3,0)\end{array}$ \\
\hline
\end{tabular}

@ Los OR se calcularon tomando como referencia la categoría de IG 18 a 23 meses. Todos los resultados están ajustados por edad materna, estado civil, tabaquismo, altura, nivel socieconómico, peso del nacimiento previo y cesárea previa.

Conclusión: EI IG menor de seis meses es un factor de riesgo independiente para resultados perinatales adversos.

Fuente de financiamiento: ninguna.

\section{Comentario}

Desde mediados de la década del 70' el IG fue analizado y reportado como un factor de importancia en el resultado perinatal. Desde entonces, las publicaciones en este sentido fueron creciendo rápidamente..$^{1-4} \mathrm{La}$ asociación ente IG cortos y mortalidad infantil es invariable, e independiente de la edad de la madre, la paridad y otros factores de confusión*, y no podría ser explicada por diferencias socioeconómicas. ${ }^{2,5-9}$ Numerosos estudios han sugerido que intervalos IG menores a 12 meses se asocian a resultados perinatales desfavorables; y que dicho riesgo se incrementa con la disminución de su duración. ${ }^{2.4}$ Sin embargo los múltiples factores de confusión* actuantes debilitan las conclusiones. En Latinoamérica, como en otras regiones no desarrolladas, la interrelación entre IG y factores sociodemográficos es compleja. Las mayores frecuencias se observan en mujeres jóvenes de bajo nivel socioeconómico o en mayores de 35 años que posponen la maternidad y tienen un elevado nivel social..$^{1,2,7}$ Tomando modelos utilizados para explicar y predecir patrones de fertilidad en las poblaciones, algunos epidemiólogos han planteado que el IG actuaría como una variable

\section{Dr. Hugo Krupitzky [S Servicio de Obstetricia CEMIC.}

intermedia entre el resultado adverso, la mortalidad infantil, y determinantes ambientales, conductuales y biológicos. ${ }^{1,2,7}$ Este planteo resulta muy interesante pues es más fáctico pensar en intervenciones de salud que modifiquen el IG como la anticoncepción, que en acciones destinadas a modificar condiciones sociales, biológicas o demográficas desfavorables. De hecho se ha estimado que si los nacimientos en Latinoamérica ocurriesen luego de 27 y hasta 32 meses de uno previo, la mortalidad perinatal disminuiría en un $14 \%$, lo que equivale a una reducción de 60,500 muertes anuales. ${ }^{10}$

\section{Conclusiones de comentador}

Si bien la relación entre IG corto y efectos adversos neonatales es compleja y obedece a distintas causas, focalizarse en la misma y aumentando el intervalo como por ejemplo a través de la anticoncepción, podría disminuir la mortalidad perinatal.

Krupitzky H. Un intervalo intergestacional menor a seis meses se asocia con mayor riesgo de parto pretérmino y muerte neonatal. Evid. actual. práct. ambul. 2005;8:77. Comentado de Smith G, Pell J, Dobbie R. Interpregnancy interval and risk of preterm birth and neonatal death, a retrospective cohort study. BMJ 2003; 327: 313. PMID: 12907483

\section{Referencias:}

1. Bongaarts, J. A framework for analyzing the proximate determinants of fertility. Pop. Develop Rev. 1978; 4:105 -32

2. Park, C; Siasakul, S; Saengtienchai, C. Effect of birth spacing on infant survival in Thailand: Two-stage logit analysis. S. Asian J Trop Med Public Health. $1994 ; 25: 50-9$

3. Yerushalmy, J. Bierman, JM, Kempk, DH. Longitudinal study of pregnancy on the island of Kauai Territory of Hawaii: analysis of previous reproductive history. Am. J Obstet Gynecol. 1956: 71: $80-8$

4. Easman NJ. The effect of interval between births on maternal and fetal outlook. Am J Obst Gynecol. 1944; 47: 445-66

5. Huttly SR, Victora CG, Barros FC, Vaughan JP. Birth spacing and child health in urban Brazilian children. Pediatrics 1992;89:1049-54

6. Palloni A, Aguirre GP, Lastiri S. The effects of breast-feeding and the pace of childbearing on early childhood mortality in Mexico. Bull Pan Am Health Organ 1994;28(2):93-111

7. Park CB. The place of child spacing as a factor in infant mortality: A recursive model. Am J Public Healt. 1986; 76:995-9

8. Thapa S, Short RV, Totts. Breastfeeding, birthspacing and their effect on child survival. Nature 1989; 335:679-83

9. Winikoff B. The effect of birth spacing on child and maternal healt. Fam Plann. 1983; 14:231 -245

10. Agustin CondeAgudelo, José M Belizán Maternal morbidity and mortality associated with interpregnancy interval: cross sectional study BMJ 2000;321: $1255-9$ 